\title{
Guest Editor's Comments
}

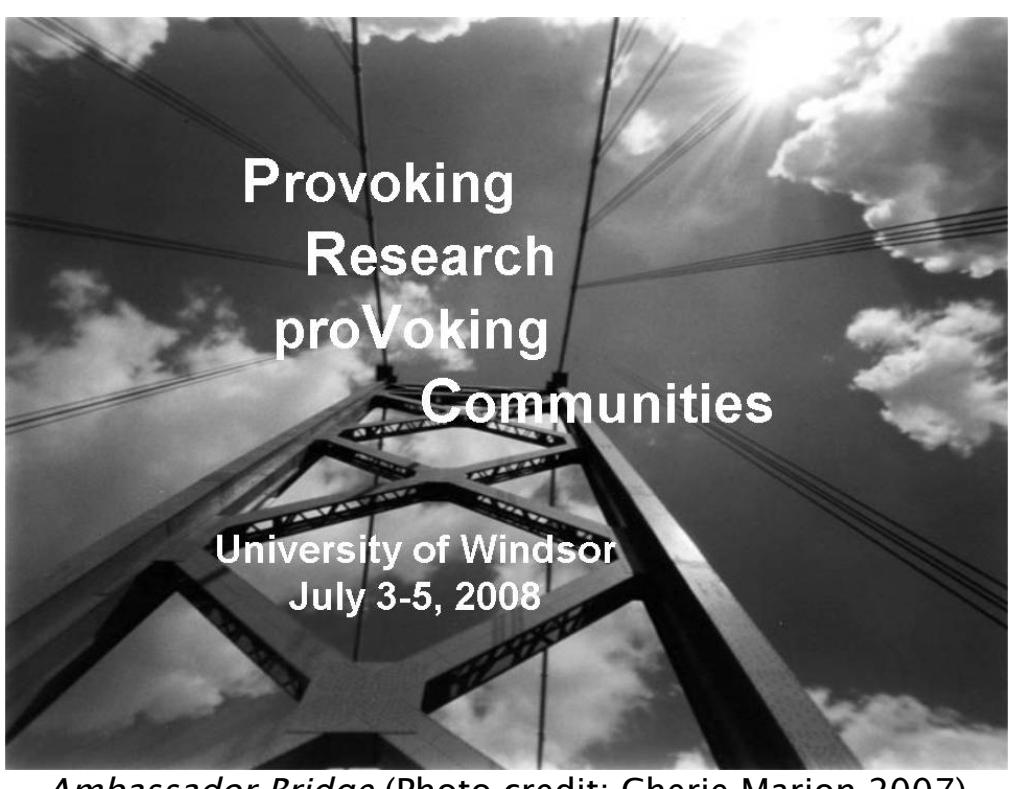

Ambassador Bridge (Photo credit: Cherie Marion 2007)

The genesis of this issue goes back several years. Darren Stanley, at the time an assistant professor at the University of Windsor and the editor of the JTL, proposed holding a conference that would stretch the boundaries of research paradigms by inviting researchers to present provoking ideas; to present in ways that were provoking; and to present research that crossed the often artificial boundary between academia and community. That initial idea did not quite take flight due to the usual mundane obstacles of logistics and financing. The following year, a committee was struck under the leadership of Nombuso Dlamini, and we built on Darren's idea. The conference took place in the spring of 2008. I was a member of the organizing committee and I wrote the call for papers that we sent out. It read, in part:

The solitary nature of much academic work - the strong borders that traditionally divide discipline from discipline, researcher from researcher, teaching from researching, work from not-work - fosters a lingua franca of textual practices, a way of talking and writing that will count as scholarly, that will be recognized by peers as 'academic'. These practices flatten the landscape of academia, and restrain attempts to disturb and erupt from within or without.

We encourage researchers to present collaboratively and in provocative formats. This could include paper and poster sessions, readers' theatre, improvisation, multi-media, or visual art forms. Presenters are invited to portray their work from multiple perspectives that include applying new methods to meet the research needs of their communities.

We hope and trust that new ideas and creative approaches will trigger conversations and build bridges between people and institutions, fields of research and the streams that run through them. 
The response was enthusiastic. We had three full days of presentations, including the plenary addresses of Chet Bowers, whose paper, The Task Facing Educational Reformers: Making the Transition from Individual Intelligence to Ecological Intelligence, leads off this special issue; Patti Lathers, who spoke about Research as Praxis; and Handel Wright, on Positive Propaganda for the New Paradigm War.

The papers that were subsequently submitted to the JTL for review and consideration for a special issue dedicated to the conference presented a challenge-we sent them out for blind review without any context for the reviewers. As a result, many reviewers were 'provoked'; sometimes delighted, sometimes maddened, by the un-traditional approach to writing and/or methodology. As editor, I read the reviewers comments and assessments while keeping in mind a context that was not available to them-a subtext of subversive practice that the conference had actively recruited.

The papers that follow provide an education in provocation. Chet Bowers challenges us to question educational traditions based on Western philosophy and ideology and to resist "liberal-progressive thinking" that has produced a consumer-dependent culture. Bowers encourages us to look to the Third World for a different model of progress, one that values intergenerational relationships, that invests in local culture and experiential knowledge and that leads to "ecological intelligence."

Kristi Buccieri, Campbell McDermid, both PhD students, and Joy Mannette, a professor, investigate the relationship between doctoral students and their supervisors as one of sorcerer and apprentice. While using autoethnography as their methodology, they produce a "polyvocal" text: a conversation, a debate, a collage of multiple voices that creates a textual space somewhere between Socratic discourse and theatre.

Joanne Elvy revisits Cuba 1961 through text and image and presents "a multiplicity of readings in the deliberate disruption of linear flow that might be akin to a theatre piece." While Elvy provides an academic account of her research, of interviews with women who were in their teens during the Cuban revolution, the text is "interrupted" by photos of the women as they are now, fifty years later, and by excerpts of the women's stories arranged as poetry. The result is to continually unsettle the reader from a linear reading of academic research.

Holly Stack-Cutler explores the challenges facing the traditional researcher when undertaking community-based research. Many of the assumptions of academic research must be reconsidered and realigned, including how research is funded, ethically reviewed, implemented, and disseminated. This article delves into the multiple roles that may be required of the community-based researchercollaborator, relationship-builder, teacher, learner-as the author describes how she negotiated her roles and her research goals with a community research partner.

Hyeran Park and Zuochen Zhang review the experimental research methods that have been used to assess the adoption of Information and Communication Technologies (ICT) in the classroom, and then discuss the use of design research as an alternative and preferable methodology for studies in "messy" classrooms. They suggest that the experimental research method of controlled study with analysis of a small number of variables produces results that have limited 
application to the classroom. This article, like Stack-Cutler's, also alludes to the constraint that academic researchers may feel to stay within the boundaries of institutionally sanctioned research paradigms in order to receive funding and gain tenure. Park and Zhang demonstrate that design research can produce reliable findings that are transferable beyond the particular research context, by carefully tailoring method to context, and through sophisticated data analysis.

Dragana Martinovic, Jelena Magliaro, and Timothy Pugh present stories of "success" and "ruin" surrounding the use of ICT within school settings. Their primary data source is a school teacher who writes stories about his use of technology. The authors analyse two of his narratives to determine relationships, incidents of rejection, conflict, resolution, and success. They construct a time-line of "encounters" which creates a narrative of analysis. Their paper provides a model for "reaching across narrative space" to build productive and mutually beneficial collaborations between researchers and practitioners.

Final, Terry Sefton (guest editor of this issue) and Sheila Windle describe how they developed a multimedia "movie" to convey the nature as well as the findings of an action research project. Sheila Windle was part of a research team that worked with teams of classroom teachers over two years during a provincewide project funded by the Elementary Teachers' Federation of Ontario. This paper describes how Windle and Sefton developed a way of presenting and disseminating findings, using multimedia as a vehicle for revisiting and reflecting on the "cyclical," "non-linear," "multi-layered," and "context-specific" character of action research.

In briefly summarizing the papers I am struck by two things: that "provoking research" may be provoking because of its approach-its tools of data collection, data analysis, writing, or dissemination - or because of its concepts. And that "provoking communities" may happen in a number of ways: by challenging academic institutions of education and funding to think differently about "acceptable" research methodologies; by provoking researchers to think differently about the tools they use and the relations they build with research partners; and by provoking community partners to reconsider the "gap" between academic research and local problems and to take an active and dynamic role in research that will benefit community interests.

I am grateful to Darren Stanley for getting all of this started with his big idea of a "provoking" conference; for the work of Nombuso Dlamini in taking it forward and realizing the dream; to all who took part in the conference; to those who provided the Journal of Teaching and Learning with their thoughtprovoking papers; and to all of you who are actively pursuing the goals of provoking research and provoking communities.

Terry Sefton

Guest Editor 